\title{
The microRNA-218 and ROBO-1 signaling axis correlates with the lymphatic metastasis of pancreatic cancer
}

\author{
HANG HE ${ }^{1}$, YANG DI ${ }^{1}$, MINRUI LIANG ${ }^{2}$, FENG YANG $^{1}$, LIE YAO $^{1}$, SIJIE HAO ${ }^{1}$, \\ $\mathrm{JI} \mathrm{LI}^{1}$, YONGJIAN JIANG ${ }^{1}$, CHEN JIN ${ }^{1}$ and DELIANG FU ${ }^{1}$ \\ ${ }^{1}$ Pancreatic Disease Institute, Department of Pancreatic Surgery, Huashan Hospital; ${ }^{2}$ Division of Rheumatology, \\ Shanghai Medical College, Huashan Hospital, Fudan University, Shanghai 200040, P.R. China
}

Received January 17, 2013; Accepted March 15, 2013

DOI: $10.3892 / o r .2013 .2516$

\begin{abstract}
Pancreatic cancer is known for its poor prognosis and early lymphatic metastasis is a notable characteristic. microRNAs (miRNAs) have been shown to be involved in the initiation and progression of pancreatic cancer. We, therefore, established a screening strategy to find miRNAs related to the lymphatic metastasis of pancreatic cancer and explored the target genes of miRNAs. miRNA array profiles were analyzed in tissue samples [pancreatic ductal adenocarcinoma (PDAC) and matched adjacent benign tissues (MAT)] and cell lines (BxPC-3-LN and BxPC-3). Combined analysis of profiling data from tissue samples and cell lines was used to identify miRNAs related to the lymphatic metastasis of pancreatic cancer. The expression levels of miRNAs were confirmed by real-time reverse transcription PCR (RT-PCR) in tissue samples and cell lines. The correlation between miRNAs and clinicopathological characteristics was investigated. The expression features of miRNAs in pancreatic cancer, precursor lesions and metastatic lymph nodes were characterized by in situ hybridization (ISH). Predicted target genes of miRNAs were validated by RT-PCR and the protein levels of target genes were revealed by western blotting. Seventy and 63 miRNAs were differentially expressed in pancreatic cancer and BxPC3-LN, compared to MAT and BxPC-3, respectively. Combined microarray analysis found 4 co-differentially expressed miRNAs (miRNA-663, miRNA-145, miRNA-218 and let-7) related to the lymphatic metastasis of pancreatic cancer. miRNA-218 was significantly downregulated in BxPC-3-LN (fold-change $>10$ ) and the expression levels of miRNA-218 were confirmed by RT-PCR. The group with lymph node metastasis and the elder group (age >64) showed lower expression of miRNA-218 ( $\mathrm{P}=0.003$ and 0.002$)$, compared to patients without lymph nodes metastasis and patients in the younger
\end{abstract}

Correspondence to: Professor Deliang Fu, Pancreatic Disease Institute, Department of Pancreatic Surgery, Huashan Hospital, Fudan University, 12 Middle Wulumuqi Road, Shanghai 200040, P.R. China E-mail: surgeonfu@yahoo.com.cn

Key words: microRNA, pancreatic cancer, lymphatic metastasis group (age $\leq 64$ ), respectively. The expression of miRNA-218 showed a decreasing trend from normal acinar/ductal epithelium, intraductal papillary mucinous neoplasm (IPMN), pancreatic cancer to metastatic lymph nodes by ISH. Among 8 predicted target genes of miRNA-218, rodent bone (ROBO-1) was confirmed to be upregulated in both mRNA and protein levels in pancreatic cancer. In conclusion, we established a screening strategy based on microarray results and found miRNA-218 to be a notable gene related to lymphatic metastasis of pancreatic cancer. Downregulation of miRNA-218 and upregulation of ROBO-1 were first demonstrated in pancreatic cancer. The miRNA-218 and ROBO-1 signaling axis may contribute to the lymphatic metastasis of pancreatic cancer.

\section{Introduction}

Pancreatic cancer is the fourth leading cause of cancer-related mortality and patients suffer a poor prognosis with a total 5 -year survival rate $<5 \%$ among all major types of cancer (1). Surgical resection is the only curative treatment for pancreatic cancer; however, extensive local invasion and early metastasis to regional lymph nodes or other sites in pancreatic cancer lead to unresectable diseases. Therefore, molecules which are involved in tumor metastasis are critical for staging and may be unique therapeutic targets in pancreatic cancer.

miRNAs are small non-coding RNAs, approximately 20-25 nucleotides in length. They lead to inhibition of translation or direct degradation of their target mRNAs by binding to the 3'UTR regions of these mRNAs, which participate in various biological processes, such as proliferation, apoptosis and cell differentiation. miRNAs also play a role in the progression of tumor and are termed as oncomiR or anti-oncomiR. In pancreatic cancer, investigators have found some miRNAs, including miR-96, miR-217, let-7 and miR-34 (2-5), that serve as tumor suppressors, and another class of miRNAs, including miR-21, miR-155 and miR-200 family members (6-9), that promote tumor progression.

Previous studies have reported their miRNA profiling results (10-12) and the majority are involved in tumor growth, apoptosis, invasion and chemotherapy resistance (9,13-18); however, miRNAs related to lymphatic metastasis of pancreatic cancer have yet to be fully clarified. According to the miRNA array profiling of both lymphatic metastasis cell lines 
and clinical tissue samples, we investigated the gene related to lymphatic metastasis of pancreatic cancer and explored its target genes.

\section{Materials and methods}

Cell lines. The human pancreatic cancer cell lines BxPC-3, SW1990 and Panc-1 were obtained from the Cell Library of the Chinese Academy of Sciences. BxPC-3-LN was a human pancreatic cell line with relative pure nature of lymphatic metastasis, which was derived from a metastatic lymph node of an ectopically implanted tumor model described in our previous studies $(19,20)$. Briefly, we implanted the parental cell line BxPC-3 $\left(10^{6}\right.$ cells) in the foot pads of nude mice (BALB/C) and harvested the metastatic lymph nodes, followed by primary culturing of the tumor cells. We repeated the procedure and purified the nature of the lymphatic metastasis of the cell line BxPC-3 and found BxPC-3-LN. BxPC-3 and BxPC-3-LN were cultured in RPMI-1640 medium (Thermo Fisher Scientific, Shanghai, China) supplemented with $10 \%$ fetal bovine serum (FBS; Gibco, Grand Island, NY, USA). SW1990 and Panc-1 were cultured in Leibovitz's L-15 medium (Gibco) and DMEM medium (Thermo Fisher Scientific) supplemented with $10 \%$ FBS, respectively. All cell lines were maintained at $37^{\circ} \mathrm{C}$ with $5 \% \mathrm{CO}_{2}$.

Clinical tissue samples. Patients with pancreatic tumors who received curative resections of tumors at the Department of Pancreatic Surgery, Huashan Hospital, between 2009 and 2011, were enrolled in the present study. For microarray analysis, PCR and western blotting, clinical tissue samples from the resected pancreatic tumors were snap frozen in liquid nitrogen and stored at $-80^{\circ} \mathrm{C}$ until the extractions of RNA and protein. Sections adjacent to the fresh frozen tissue blocks were used to confirm the diagnosis of pancreatic ductal adenocarcinoma (PDAC) by two experienced pathologists. A total of 98 fresh frozen tissue samples from 61 patients consisted of 6 PDAC samples with matched adjacent benign tissues (MAT), 51 PDAC samples and 35 unmatched adjacent benign tissue samples. For in situ hybridization, paraffin-embedded tissue samples included 10 PDACs, 3 intraductal papillary mucinous neoplasms (IPMNs), 4 metastatic lymph nodes and 13 MATs from 13 patients. All procedures were performed with the written informed consent of patients and in accordance with the policies and practices of the Institution's Ethics Committee. Tumor stage was evaluated according to the American Joint Committee on Cancer (AJCC) Cancer Staging, 6th edition. Clinical data of the patients were collected.

miRNA microarray analysis. miRNAs were extracted from cells and tissues using the mirVana ${ }^{\mathrm{TM}}$ miRNA Isolation kit (Applied Biosystems, p/n AM1556) following the manufacturer's instructions. The quality control criteria of RNA were $\mathrm{RIN} \geq 6.0,28 \mathrm{~S} / 18 \mathrm{~S}>0.7$ and A260/A280>1.8 (Agilent 2100 Bioanalyzer). Human miRNA microarray (Agilent, version 14.0 and illumina miRNA array) was used to examine the differential expression profile of miRNAs in groups BxPC-3 vs. BxPC-3-LN and PDAC vs. MAT (6 paired fresh frozen tissue samples). Briefly, the RNA sample was dephosphorylated with calf intestinal phosphatase, degenerated with DMSO, labeled
Table I. Primer sequences for RT-PCR.

\begin{tabular}{ll}
\hline Gene & \multicolumn{1}{c}{ Sequence $\left(5^{\prime} \rightarrow 3^{\prime}\right)$} \\
\hline BIRC5 & F: ACTTGGCCCAGTGTTTCTTCT \\
& R: TCTTGGCTCTTCTCTGTCCA \\
EGFR & F: AGCCTCCAGAGGATGTTCAA \\
& R: GGAATTCGCTCCACTGTGTT \\
PAXILLIN & F: ACGTCTACAGCTTCCCCAACAA \\
& R: AGCAGGCGGTCGAGTTCA \\
CETN2 & F: GGCTTTGAACCCAAGAAAGA \\
& R: TTTGAACGAAATCTTCCAGTT \\
LAMB3 & F: GGCCTGCTATCCACCTGTT \\
& R: GCCACATTCTCTACTCGGTGA \\
HOXA1 & F: AGCCACCAAGAAGCCTGTC \\
& R: TCCTTCTCCAGTTCCGTGAG \\
LEF1 & F: GATCAGTCATCCCGAAGAGG \\
& R: GTGTTCTCTGGCCTTGTCGT \\
ROBO-1 & F: GCGTGCAGTACTAAGGGAACA \\
& R: GGCTTCTTACATGAACATAATGAA
\end{tabular}

with T4 RNA ligase and cyanine-3-pCp, hybridized at $55^{\circ} \mathrm{C}$ using Microarray Hybridization Chamber and Hybridization oven, washed and scanned. Array-comparative genomic hybridization (array-CGH) analysis was carried out and the differential extents of miRNAs between groups were denoted as Diff Pval, Diff Score or fold-change.

$R T-P C R$. The expression levels of miRNA and mRNAs were quantitatively analyzed by RT-PCR in cells and fresh frozen tissue samples. miRNAs and mRNAs were extracted from cells and tissues using TRIzol (Invitrogen, Carlsbad, CA, USA). The purity and concentration of RNA were evaluated by A260/A280 using NanoDrop ND-1000 Spectrophotometer (Thermo Fisher Scientific, Courtaboeuf, France). miRNAs and mRNAs were converted to cDNAs using the TaqMan ${ }^{\circledR}$ MicroRNA Reverse Transcription kit (Applied Biosystems, Foster City, CA, USA) and the PrimeScript RT Master Mix Kit (Takara, Dalian, China) respectively, by Mastercycler (Eppendorf, USA). The real-time PCR was performed by Applied Biosystems 7500 Real-Time PCR System, using TaqMan MicroRNA assays (Applied Biosystems) for miRNAs and SYBR Premix Ex Taq II (Takara) for mRNAs, respectively, following the manufacturer's instruction. U6 snRNA and GAPDH were selected for normalization to the expression levels of miRNAs and mRNAs. Relative quantitations (RQs) of miRNAs and mRNAs were calculated using the $2^{-\Delta \Delta C t}$ method. The sequences of RT-PCR primers are listed in Table I.

In situ hybridization (ISH). Double-DIG labeled locked nucleic acid microRNA probe, complementary to hsa-miR-218 (P/N AM17100; Exiqon, Vedbaek, Denmark) was designed for ISH of 13 paired paraffin-embedded PDAC and MAT samples, conforming to the manufacturer's instructions. The scrambled microRNA probe was used as negative control. In 


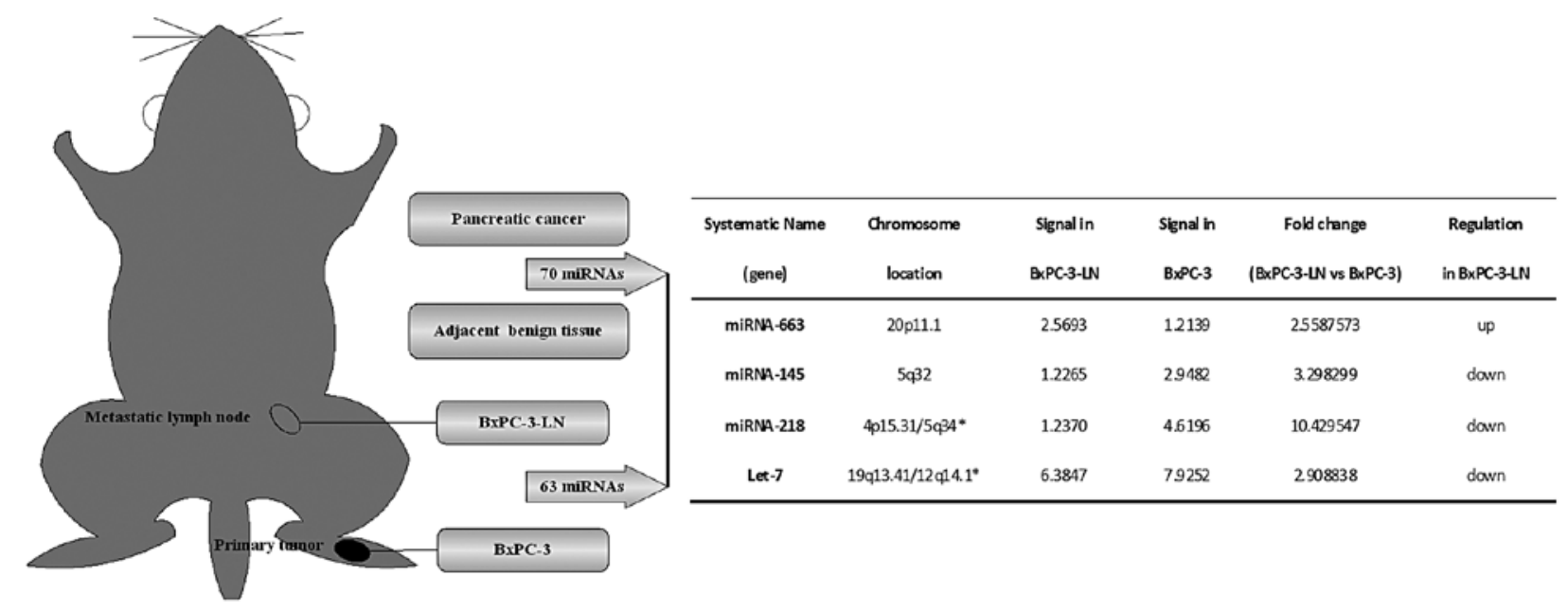

Figure 1. Differentially expressed miRNAs in cell lines and tissue samples of profiling results. Four miRNAs were related to the lymphatic metastasis of BXPC-3-LN and human pancreatic cancer (asterisk indicates the same mature products).

addition, 4- $\mu \mathrm{m}$ tissue sections were deparaffinized in xylene and ethanol, incubated with Proteinase-K $(15 \mu \mathrm{g} / \mathrm{ml}, 10 \mathrm{~min}$ at $\left.37^{\circ} \mathrm{C}\right)$, washed twice in PBS, dehydrated in ethanol, hybridized $\left(1 \mathrm{~h}\right.$ at $\left.49^{\circ} \mathrm{C}\right)$ with hybridization mix (1:500 dilution of probe), washed in standard saline citrate buffer, and blocked. Subsequently, staining was developed with sheep antidigoxin-horseradish peroxidase antibody and visualized with diaminobenzidine followed by hematoxylin counter staining of nuclear. The results were evaluated independently by two experienced pathologists. In brief, 10 HPFs of each section were chosen randomly. Staining intensity was ranked as negative staining (0), weak staining (1+) and strong staining (2+). The mean positive-staining cells (PSCs) were scored as 0 (PSC <1\%), 1 (PSC 1-25\%), 2 (PSC 25-50\%), 3 (PSC >50\%).

Target genes of miRNA. Eight genes (BIRC5, EGFR, PAXILLIN, CETN2, LAMB3, HOXA1, LEF1 and ROBO-1) which were predicted by biological databases (www. targetscan.org and pictar.mdc-berlin.de) and reported to be the target genes of miRNA-218 in various tumors in the literature, were validated in pancreatic cancer. The transcriptional levels of theses target genes were investigated in PDAC and adjacent benign tissue by RT-PCR.

Western blotting. Protein from fresh frozen tissue samples was extracted and quantified. Western blotting was performed according to the standard procedure. The primary antibody, antiRobo-1 (rabbit polyclonal antibody, sc-25672) was purchased from Santa Cruz Biotechnology (Santa Cruz, CA, USA). GAPDH (rabbit monoclonal antibody, 2251-1) was purchased from Epitomics (Burlingame, CA, USA) and used for control. Chemiluminescent substrate (34079; Thermo Fisher Scientific, Rockford, IL, USA) was used for detection of immunoblots. The images were developed by Fujifilm Las-3000.

Statistical analysis. Measurement variable was summed as means \pm SD and compared by the Student's t-tests, unless otherwise indicated. SPSS 16.0 software was employed for statistical analysis and $\mathrm{P}<0.05$ was considered to indicate a statistically significant difference.

\section{Results}

Differential expression of miRNAs in array-CGH analysis. Profiling analysis of group (PDAC and MAT) was performed to examine the differential miRNAs in pancreatic cancer, which may be involved in several important processes of tumor biology. Seventy miRNAs were found differentially expressed $(\mathrm{P}<0.05$ and Diff Score $>13$ or $<13)$, including 43 downregulated miRNAs and 27 upregulated miRNAs in pancreatic cancer compared to adjacent benign tissue. Differential expression of miRNAs between the cell lines BxPC-3 and BxPC-3-LN were also characterized by array$\mathrm{CGH}$, which may include genes responsible for lymphatic metastasis of pancreatic cancer. Sixty-three miRNAs differentially expressed in BxPC-3-LN compared to BxPC-3, including 33 downregulated miRNAs and 30 upregulated miRNAs. Combined analysis of tissue-based profiling and cell-based profiling results revealed 4 co-differentially expressed miRNAs (Fig. 1). Among these co-differentially expressed miRNAs, miRNA-218 was the most notable gene (fold-change $>10$ ) in BxPC-3-LN.

Downregulation of miRNA-218 in pancreatic cancer. Quantitative real-time PCR was performed in 37 PDAC samples, 35 adjacent benign tissue samples and cell lines to confirm the expression level of miRNA. miRNA-218 was found downregulated in pancreatic cancer and the cell line BxPC-3-LN (Fig. 2). In tissue samples, the RQ of miRNA-218 was significantly lower $(\mathrm{P}=0.001)$ in pancreatic cancer $(0.0684 \pm 0.0339)$ than in benign tissues $(0.2055 \pm .0208)$. In cell lines, the RQ of miRNA-218 was $1.0005 \pm 0.0429$ in BxPC-3-LN and $1.4961 \pm 0.0362$ in BxPC-3 $(\mathrm{P}<0.001)$. Furthermore, the cell lines SW1990 and Panc-1 showed higher RQ of miRNA-218 $(26.9468 \pm 2.1649$ and $9.8628 \pm 0.4647$, respectively) compared to BxPC-3-LN5 $(\mathrm{P}<0.001)$.

Expression of miRNA-218 and clinicopathological characteristics. The relationship between the expression levels of miRNA-218 and clinicopathological data of PDAC patients was analyzed in a total of 37 PDAC cases (Table II). The 
Table II. Relationship between clinicopathological characteristics and miRNA-218 expression in PDAC.

\begin{tabular}{|c|c|c|c|}
\hline Clinicopathological characteristics & No. of patients (37) & RQ of miRNA-218 & P-value \\
\hline \multicolumn{4}{|l|}{ Gender } \\
\hline Male & 26 & $0.0747 \pm 0.0552$ & \multirow[t]{2}{*}{$0.207^{\mathrm{a}}$} \\
\hline Female & 11 & $0.0533 \pm 0.0495$ & \\
\hline \multicolumn{4}{|l|}{ Age (years) } \\
\hline$>64$ & 17 & $0.0414 \pm 0.0290$ & \multirow[t]{2}{*}{$0.002^{\mathrm{a}, \mathrm{b}}$} \\
\hline$\leq 64$ & 20 & $0.0912 \pm 0.0599$ & \\
\hline \multicolumn{4}{|l|}{ Stage } \\
\hline I & 11 & $0.0949 \pm 0.0691$ & \multirow[t]{5}{*}{$0.348^{\mathrm{c}}$} \\
\hline IIA & 6 & $0.0456 \pm 0.0246$ & \\
\hline IIB & 19 & $0.0607 \pm 0.0480$ & \\
\hline III & 1 & 0.0585 & \\
\hline IV & 0 & & \\
\hline \multicolumn{4}{|l|}{ Differentiation grade } \\
\hline I-II & 20 & $0.0613 \pm 0.0526$ & \multirow[t]{2}{*}{$0.610^{\mathrm{a}}$} \\
\hline III & 16 & $0.0692 \pm 0.0481$ & \\
\hline \multicolumn{4}{|l|}{ Size $(\mathrm{cm})$} \\
\hline$\leq 3$ & 21 & $0.0684 \pm 0.0487$ & \multirow[t]{2}{*}{$0.602^{\mathrm{a}}$} \\
\hline$>3$ & 16 & $0.0683 \pm 0.0616$ & \\
\hline \multicolumn{4}{|l|}{ Lymph node metastasis } \\
\hline Positive & $16(20)$ & $0.0398 \pm 0.0154$ & \multirow[t]{2}{*}{$0.003^{\mathrm{a}, \mathrm{b}}$} \\
\hline Negative & $14(17)$ & $0.0912 \pm 0.0589$ & \\
\hline \multicolumn{4}{|l|}{ Vascular involvement } \\
\hline Positive & 9 & $0.0527 \pm 0.0468$ & \multirow[t]{2}{*}{$0.288^{\mathrm{a}}$} \\
\hline Negative & 28 & $0.0734 \pm 0.0557$ & \\
\hline \multicolumn{4}{|l|}{ Perineural invasion } \\
\hline Positive & 17 & $0.0728 \pm 0.0576$ & \multirow[t]{2}{*}{$0.648^{\mathrm{a}}$} \\
\hline Negative & 20 & $0.0645 \pm 0.0516$ & \\
\hline \multicolumn{4}{|l|}{ Neoadjuvant chemotherapy } \\
\hline Yes & 6 & $0.0733 \pm 0.0419$ & \multirow[t]{2}{*}{$0.484^{\mathrm{a}}$} \\
\hline No & 31 & $0.0674 \pm 0.0564$ & \\
\hline
\end{tabular}

${ }^{\mathrm{a}}$ Mann-Whitney; ${ }^{\mathrm{b}}$ statistical significance $<0.05$; ${ }^{\mathrm{K}}$ Kuskal-Wallis. PDAC, pancreatic ductal adenocarcinoma. RQ, relative quantitation.

differentiation level was underdetermined in 1 case and 7 cases were without definite status of lymph nodes. The young patients (age $\leq 64$ years) showed a relatively higher expression level of miRNA-218 $(\mathrm{P}=0.002)$ than the older patients (age $>64$ years). Tumor stage tended to correlate with miRNA-218 expression ( $\mathrm{P}>0.05)$. The group with lymph node metastasis showed lower expression level of miRNA-218 than the group without $(\mathrm{P}=0.003)$. No significant differences in the expression levels of miRNA-218 were found grouped by the factors of gender, differentiation grade, tumor size, vascular involvement status, perineural invasion status and neoadjuvant chemotherapy.

ISH of miRNA-218. Locked nucleic acid ISH was performed to characterize the expressing features of miRNA-218 in pancreatic cancer. The intensity and abundance of miRNA-218 in
MAT, IPMN and PDAC was analyzed (Table III). Widespread and high expression of miRNA-218 was found in both normal acinar and ductal epithelium, whereas focal or low expression of miRNA-218 was found in PDAC. The precursor lesion of pancreatic cancer, IPMN expressed less miRNA-218 than normal acinar or ductal epithelium, but more compared to PDAC (Fig. 3). The expression features of miRNA-218 according to tumor grade revealed that the lower cell differentiation showed lower expression of miRNA-218. Almost negative expression was demonstrated in metastatic lymph nodes, compared to corresponding primary lesions in PDAC (Fig. 4).

Target genes of miRNA-218 in pancreatic cancer. Eight target genes of miRNA-218 were quantitatively analyzed in 31 PDAC samples and 13 adjacent benign tissue samples. The 
Table III. ISH of miRNA-218.

\begin{tabular}{|c|c|c|c|c|c|c|c|}
\hline & \multicolumn{3}{|c|}{ Intensity rank } & \multicolumn{4}{|c|}{ Mean PSC score } \\
\hline & 0 & $1+$ & $2+$ & 0 & 1 & 2 & 3 \\
\hline PDAC (case) & 3 & 7 & 0 & 3 & 4 & 2 & 1 \\
\hline IPMN (case) & 0 & 3 & 0 & 0 & 0 & 1 & 2 \\
\hline MAT (case) & 0 & 2 & 11 & 0 & 0 & 1 & 12 \\
\hline $\mathrm{P}$-value & \multicolumn{3}{|c|}{$<0.001$} & \multicolumn{4}{|c|}{$<0.001$} \\
\hline
\end{tabular}

ISH, in situ hybridization; PSC, mean positive-staining cells; PDAC, pancreatic ductal adenocarcinoma; IPMN, intraductal papillary mucinous neoplasm; MAT, matched adjacent benign tissues. Statistical method, Kruskal-Wallis test.

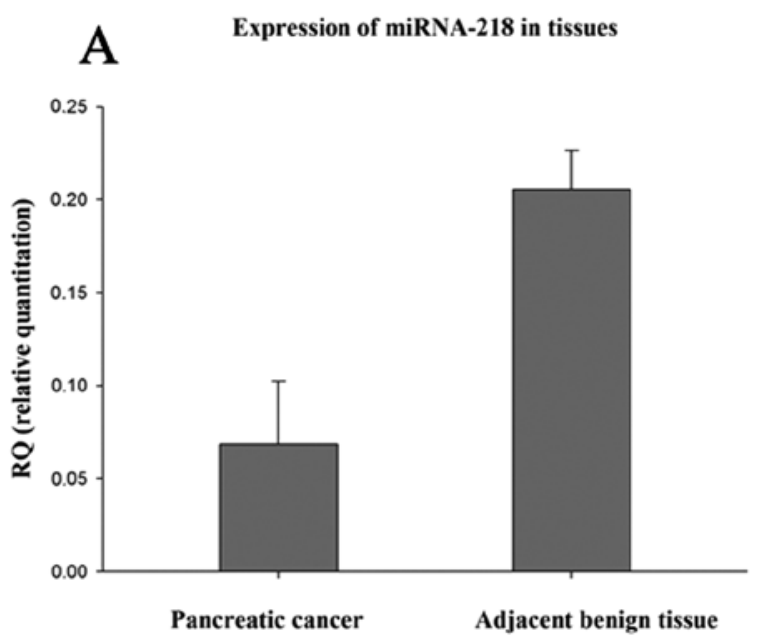

\section{B \\ Expression of miRNA-218 in cells}



Figure 2. (A) RQ of miRNA-218 in tissue samples. miRNA-218 was downregulated in pancreatic cancer compared to adjacent benign tissue. (B) RQ of miRNA-218 in cell lines. BxPC-3-LN showed lower expression of miRNA-218 than other cell lines.

expression level of ROBO-1 was found upregulated in PDAC compared to adjacent benign tissues $(\mathrm{P}=0.0066,26.75 \pm 36.60$ vs. $4.849 \pm 2.865$ ), which was consistent with the regulatory pattern of miRNA-218. BIRC5, EGFR, PAXILLIN, CETN2,

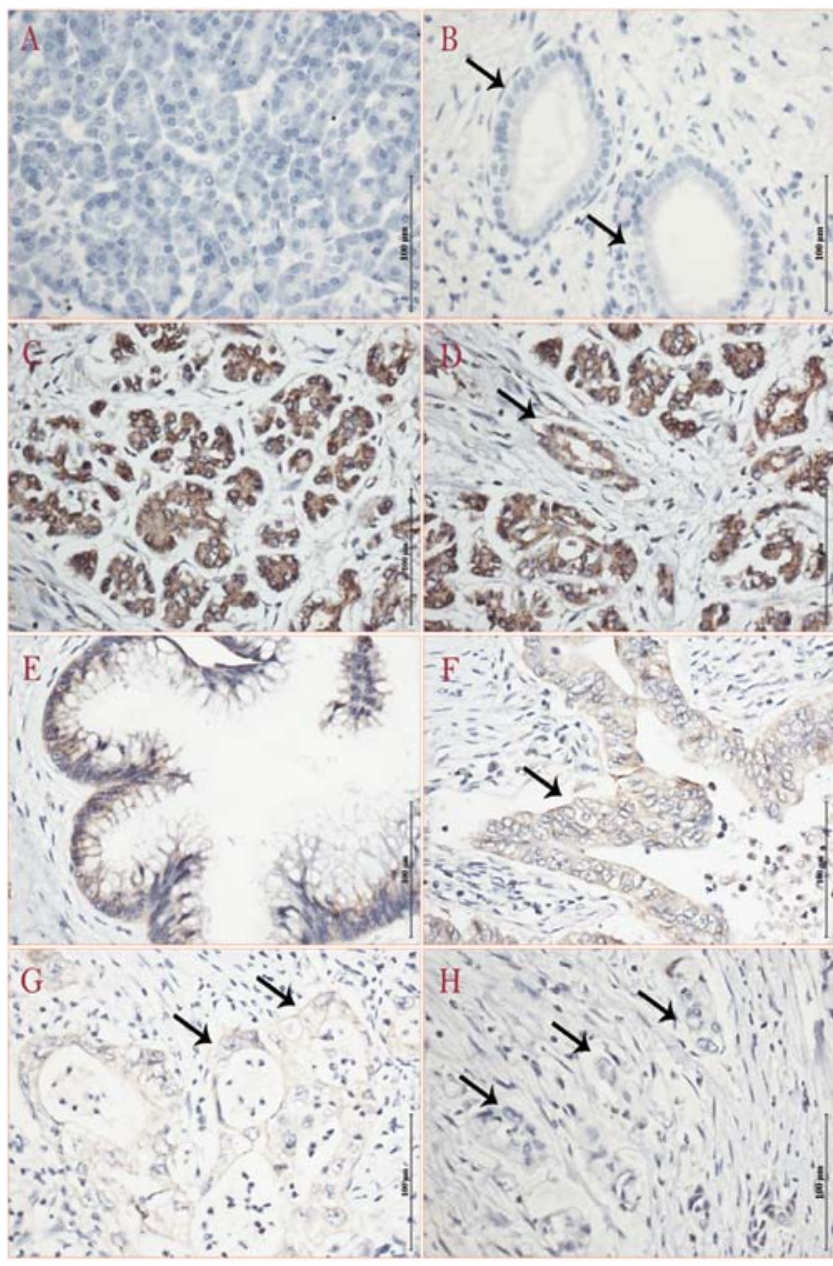

Figure 3. (A and B) Negative control of in situ hybridization in normal pancreatic acinus and duct. (C and D) Widespread and high expression (brown) of miRNA-218 were shown in normal acinar or ductal epithelium and mainly distributed in cytoplasm. (E) Intraductal papillary mucinous neoplasm showed medium expression of miRNA-218 compared to normal acinus and duct. (F, G and $\mathrm{H}$ ) Pancreatic ductal adenocarcinoma showed even lower expression of miRNA-218 and the expression level decreased as the grade increased (F:I, G:II and H:III).

LAMB3, HOXA1 and LEF1 showed no statistical significance (Fig. 5A). It was confirmed that the protein level of ROBO-1 was significantly upregulated in PDAC compared to adjacent benign tissues by western blotting (Fig. 5B). 

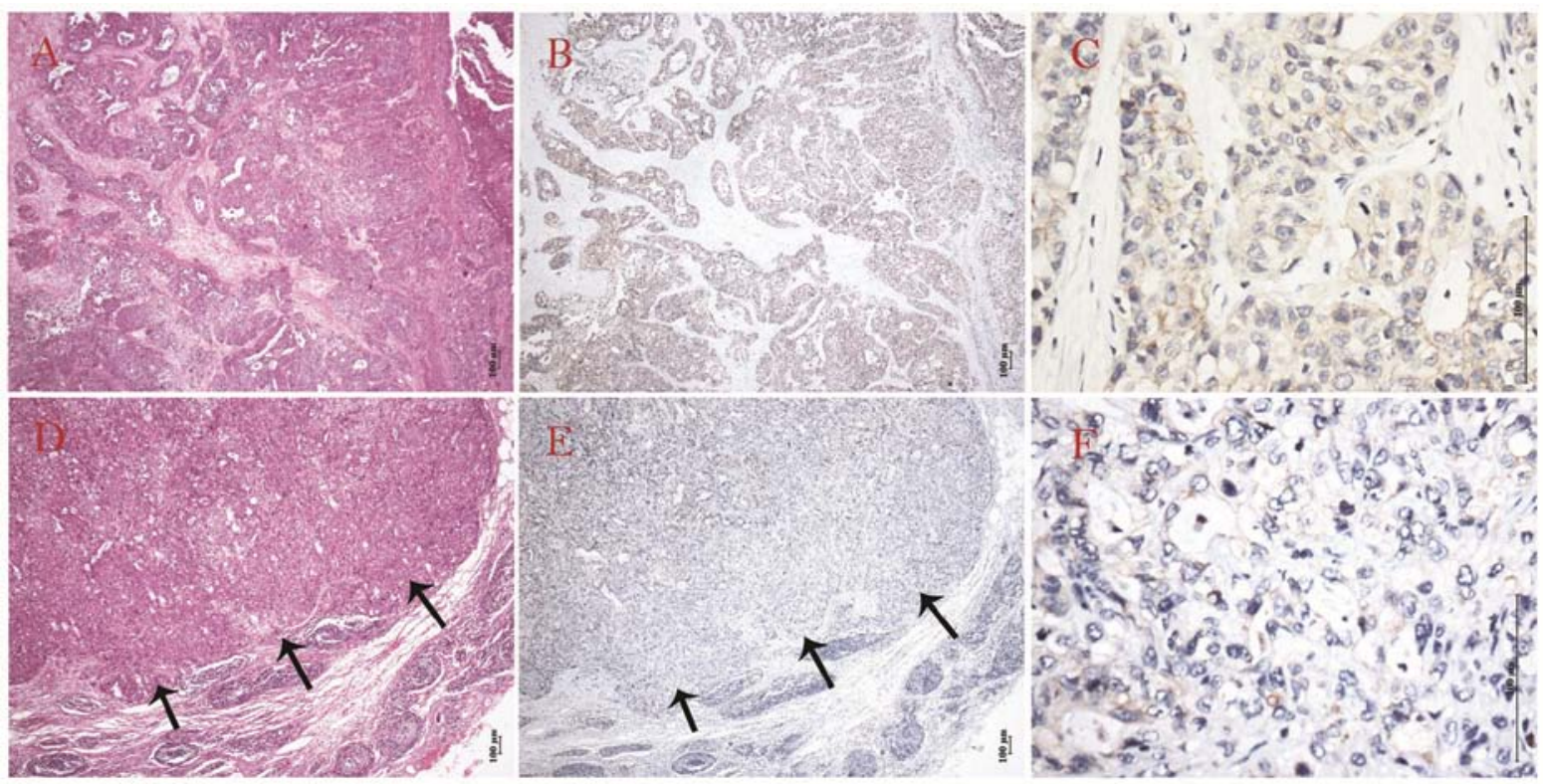

Figure 4. (A and D) Hematoxylin and eosin staining of primary and lymphatic metastasis lesions in pancreatic ductal adenocarcinoma. (B and E) In situ hybridization (ISH) of miRNA-218 in sites of primary and lymphatic metastasis lesions (original magnification, $\mathrm{x} 40$ ). Lymphatic metastasis lesion had even lower expression of miRNA-218 compared to primary lesion. (C and F) ISH of miRNA-218 in primary and lymphatic metastasis lesions (original magnification, $\mathrm{x} 400)$. Sporadic and weak expression of miRNA-218 was demonstrated in tumor cells of lymphatic metastasis lesion.

\section{Discussion}

miRNAs are a series of small non-coding RNAs and up to $30 \%$ of human genes are regulated by miRNAs. Since the first lin-4 miRNA was discovered in 1993 (21), the family of miRNAs has been highlighted and the role of miRNAs in tumor biology has also been revealed. In pancreatic cancer, pioneer studies described aberrantly expressed miRNAs $(2-3,5,9,18,22)$. Among these miRNAs, miRNA-21 and miRNA-155 are the most notable genes, which regulate proliferation, apoptosis and therapy-resistance of pancreatic cancer in principal $(7,9,18,22,23)$. Lymphatic metastasis is an unfavorable event in patients with pancreatic cancer $(24,25)$. Nevertheless, conventional profiling results cannot trace genes related to the lymphatic metastasis of pancreatic cancer. We previously established the ectopic-implanted pancreatic cancer model and found the cell line BxPC-3-LN metastasized to regional or distant lymph nodes much earlier than the parental cell line BxPC-3 $(19,20)$. The genetic heterogeneity of primary carcinoma is preserved in pancreatic cancer and clonal population that gives rise to metastasis is genetically evolved from the original parental clone (26). Therefore, we performed miRNA profiling of these two cell lines, to find the aberrantly expressed miRNAs potentially promoting lymphatic metastasis. Combined analysis of profiling results between clinical tissue samples and cell lines confirmed 4 coincident differentially expressed miRNAs. Of these 4 miRNAs, miRNA-218 was the most significantly altered gene in BxPC-3-LN compared to the parental cell line BxPC-3, which may be correlated to the lymphatic metastasis of pancreatic cancer and was also reported to be downregulated in pancreatic cancer in another profiling study (12).

We confirmed that miRNA-218 was downregulated in human PDAC and BxPC-3-LN by RT-PCR. Consistent with our results, miRNA-218 was found downregulated in several human solid tumors, including cervical carcinoma, lung cancer, gastric cancer, glioma, nasopharyngeal cancer, prostate cancer, bladder cancer and oral cancer, but it only indicated that miRNA-218 might function as a tumor suppressor in these tumors $(8,27-33)$. In the present study, BxPC-3-LN with decreased expression of miRNA-218 compared to BxPC-3 provided insight that miRNA-218 may be involved in the lymphatic metastasis of pancreatic cancer.

Moreover, we analyzed the clinicopathological characteristics of PDAC patients and found that the group with lymph node metastasis showed lower expression of miRNA-218 than the group without. This is consistent with the results in gastric cancer (34) and supports the hypothesis that miRNA-218 promotes the lymphatic metastasis of PDAC. Furthermore, we found miRNA-218 decreased in the elder age group and hypothesized that miRNA-218 might also act as a tumor suppressor, since pancreatic cancer and several other tumors usually develop in middle-aged and older patients, whose tumor suppressors are lost or mutated. However, there was a negative correlation between miRNA-218 and age in gastric and lung cancer $(34,35)$.

Results of ISH revealed that miRNA-218 is mainly expressed in normal acinar epithelium and ductal epithelium, whereas the mesenchyma seldom expressed miRNA-218. We speculate that miRNA-218 may regulate the differentiation of normal pancreatic acinar epithelium and ductal epithelium. Therefore, if the expression of miRNA-218 is impaired, it may induce aberrant differentiation of normal epithelium and even lead to carcinogenesis. Lower expression of miRNA-218 in IPMN compared to normal acinus or duct, suggested that the downregulation of miRNA-218 might be an early sign in the precursor lesions of pancreatic cancer. Additionally, PDAC showed even lower expression of miRNA-218 than IPMN 

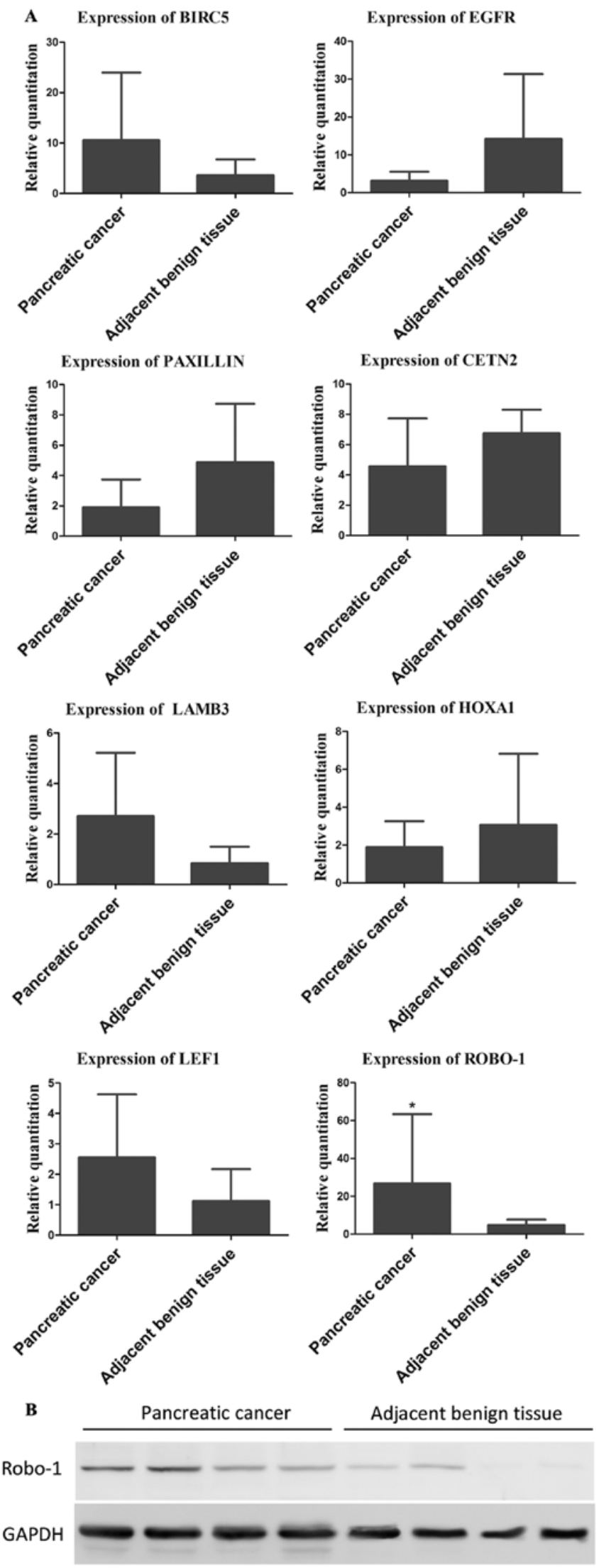

Figure 5. (A) Relative quantitation of target genes of miRNA-218 in tissue samples. The expression of ROBO-1 was significantly higher in pancreatic ductal adenocarcinoma (PDAC) compared to adjacent benign tissue $\left({ }^{*} \mathrm{P}<0.05\right)$. The expression of BIRC5, EGFR, PAXILLIN, CETN2, LAMB3, HOXA1 and LEF1 showed no significant difference between PDAC and adjacent benign tissue $(\mathrm{P}=0.3306,0.7479,0.0513,0.1650,0.1579,0.5425$ and 0.2216 , respectively). (B) Western blot result of the protein level of ROBO-1 in PDAC and adjacent benign tissue. demonstrating that the decreased expression of miRNA-218 advances as the precursor lesions transform to pancreatic cancer. Thus, the insight into the role of miRNA-218 as a marker of diagnosis or follow-up is promising. Furthermore, miRNA-218 might regulate the differentiation of tumor cells (8) and it was found that the expression of miRNA-218 was correlated with tumor grades in the present study, although it was not demonstrated by RT-PCR. Low or even negative expression of miRNA-218 in metastatic lymph nodes of PDAC was further evidence that decreased expression of miRNA-218 might promote tumor cells migrating into the lymphatic system.

A detailed mechanism of the involvement of miRNA-218 in tumor lymphatic metastasis remains to be confirmed. The target genes of miRNA-218 were hallmarks of different pathways. BIRC5, EGFR, PAXILLIN, CETN2, LAMB3, HOXA1, LEF1 and ROBO-1 were reported to be the direct targets of miRNA-218 and are involved in the carcinogenesis and tumor progression of various tumors (30,34-36). Here, we investigated these target genes of miRNA-218 in pancreatic cancer and found ROBO-1 showed higher expression of both mRNA and protein in PDAC, compared to adjacent benign tissues. ROBO-1 (Rodent Bone) which plays a novel role in bone metabolism, was first discovered by Noel et al (37) and it is a member of the emerging multifunctional uPAR/CD59/Ly-6/snake toxin family (38). It has been proved that miRNA-218 regulates ROBO-1 by binding to its 3'UTR and participates in neural development, angiogenesis and cell migration. Additionally, previous studies also found miRNA-218-ROBO-1 might promote tumor metastasis in several tumors such as nasopharyngeal cancer, gastric cancer and breast cancer $(30,34,39,40)$. Collectively, we considered that an miRNA-218-ROBO-1 dependent pathway might be involved in the lymphatic metastasis of pancreatic cancer according to the expressing features of miRNA-218 and ROBO-1 in pancreatic cancer.

In conclusion, we established a pioneer screening strategy through combined analysis of profiling results from cell lines and tissue samples, to trace the genes involved in the lymphatic metastasis of pancreatic cancer. We successfully found miRNA-218 differentially expressed in both BxPC-3-LN and pancreatic cancer which potentially promoted the lymphatic metastasis of pancreatic cancer. The downregulation of miRNA-218 and upregulation of ROBO-1 in pancreatic cancer were confirmed and we hypothesized that miRNA-218-ROBO-1 may regulate the process of metastasis, particularly lymphatic metastasis, in pancreatic cancer. The downstream mechanism of miRNA-218-ROBO-1 remains unclear and further studies regarding the lymphatic metastasis of pancreatic cancer are currently being performed.

\section{Acknowledgements}

The authors thank Feng Tang and Zunguo Du, Department of Surgical Pathology, Huashan Hospital, for their histological assistance. The study was supported by a grant from the National Natural Science Foundation of China (no. 81172274), a grant from the Shanghai Committee of Science and Technology, China (no. 11JC1401600) and a grant from the Shanghai Municipal Health Bureau, China (no. 20114v141). 


\section{References}

1. Jemal A, Siegel R, Xu JQ and Ward E: Cancer statistics, 2010 CA Cancer J Clin 60: 277-300, 2010.

2. Zhao WG, Yu SN, Lu ZH, Ma YH, Gu YM and Chen J: The miR-217 microRNA functions as a potential tumor suppressor in pancreatic ductal adenocarcinoma by targeting KRAS. Carcinogenesis 31: 1726-1733, 2010.

3. Yu SN, Lu ZH, Liu CZ, et al: miRNA-96 suppresses KRAS and functions as a tumor suppressor gene in pancreatic cancer Cancer Res 70: 6015-6025, 2010.

4. Torrisani J, Bournet B, du Rieu MC, et al: Let-7 microRNA transfer in pancreatic cancer-derived cells inhibits in vitro cell proliferation but fails to alter tumor progression. Hum Gene Ther 20: 831-844, 2009

5. Ji Q, Hao X, Zhang M, et al: MicroRNA miR-34 inhibits human pancreatic cancer tumor-initiating cells. PLoS One 4: e6816, 2009.

6. Radhakrishnan P, Mohr AM, Grandgenet PM and Hollingsworth MA: MicroRNA-200c modulates the expression of MUC4 and MUC16 in human pancreatic cancer. The 42nd Annual Meeting of the American Pancreatic Association. Pancreas 40: 1350,2011

7. Bhatti I, Lee A, James V, et al: Knockdown of microRNA-21 inhibits proliferation and increases cell death by targeting programmed cell death 4 (PDCD4) in pancreatic ductal adenocarcinoma. J Gastrointest Surg 15: 199-208, 2011.

8. Song L, Huang Q, Chen K, et al: miR-218 inhibits the invasive ability of glioma cells by direct downregulation of IKK- $\beta$. Biochem Biophys Res Commun 402: 135-140, 2010.

9. Ryu JK, Hong SM, Karikari CA, Hruban RH, Goggins MG and Maitra A: Aberrant MicroRNA-155 expression is an early event in the multistep progression of pancreatic adenocarcinoma. Pancreatology 10: 66-73, 2010.

10. Szafranska AE, Davison TS, John J, et al: MicroRNA expression alterations are linked to tumorigenesis and non-neoplastic processes in pancreatic ductal adenocarcinoma. Oncogene 26 4442-4452, 2007.

11. Lee EJ, Gusev Y, Jiang J, et al: Expression profiling identifies microRNA signature in pancreatic cancer. Int $\mathrm{J}$ Cancer 120 1046-1054, 2007.

12. Bloomston M, Frankel WL, Petrocca F, et al: MicroRNA expression patterns to differentiate pancreatic adenocarcinoma from normal pancreas and chronic pancreatitis. JAMA 297: 1901-1908, 2007.

13. Srivastava SK, Bhardwaj A, Singh S, et al: MicroRNA-150 directly targets MUC4 and suppresses growth and malignant behavior of pancreatic cancer cells. Carcinogenesis 32: 1832-1839, 2011.

14. Ohuchida K, Mizumoto K, Kayashima T, et al: MicroRNA expression as a predictive marker for gemcitabine response after surgical resection of pancreatic cancer. Ann Surg Oncol 18 : 2381-2387, 2011

15. Nakata K, Ohuchida K, Mizumoto K, et al: MicroRNA-10b is overexpressed in pancreatic cancer, promotes its invasiveness, and correlates with a poor prognosis. Surgery 150: 916-922, 2011.

16. Liffers ST, Munding JB, Vogt M, et al: MicroRNA-148a is down-regulated in human pancreatic ductal adenocarcinomas and regulates cell survival by targeting CDC25B. Lab Invest 91: 1472-1479, 2011.

17. Hao J, Zhang SY, Zhou YQ, Liu C, Hu XG and Shao CH: MicroRNA 421 suppresses DPC4/Smad4 in pancreatic cancer. Biochem Biophys Res Commun 406: 552-557, 2011.

18. Giovannetti E, Funel N, Peters GJ, et al: MicroRNA-21 in pancreatic cancer: correlation with clinical outcome and pharmacologic aspects underlying its role in the modulation of gemcitabine activity. Cancer Res 70: 4528-4538, 2010.

19. Luo GP, Yu XJ, Jin C, et al: LyP-1-conjugated nanoparticles for targeting drug delivery to lymphatic metastatic tumors. Int $J$ Pharm 385: 150-156, 2010.

20. Yang F, Jin C, Yang D, et al: Magnetic functionalised carbon nanotubes as drug vehicles for cancer lymph node metastasis treatment. Eur J Cancer 47: 1873-1882, 2011.
21. Lee RC, Feinbaum RL and Ambros V: The C. elegans heterochronic gene lin-4 encodes small RNAs with antisense complementarity to lin-14. Cell 75: 843-854, 1993.

22. Hwang JH, Voortman J, Giovannetti E, et al: Identification of microRNA-21 as a biomarker for chemoresistance and clinical outcome following adjuvant therapy in resectable pancreatic cancer. PLoS One 5: e10630, 2010.

23. Kim HR, Jang SE, Beik WH, Lee $\mathrm{SH}$ and Hwang $\mathrm{JH}$ Chemosensitivity of indole-3-carbinol via downregulation of microRNA-21 in gemcitabine-resistant pancreatic cancer cells. Asian Pacific Digestive Week, Oct, 2011. J Gastroen Hepatol 26 232, 2011

24. Massucco P, Ribero D, Sgotto E, Mellano A, Muratore A and Capussotti L: Prognostic significance of lymph node metastases in pancreatic head cancer treated with extended lymphadenectomy: not just a matter of numbers. Ann Surg Oncol 16 3323-3332, 2009

25. Riediger H,Keck T, Wellner U, et al: The lymph node ratio is the strongest prognostic factor after resection of pancreatic cancer. J Gastrointest Surg 13: 1337-1344, 2009.

26. Yachida S, Jones S, Bozic I, et al: Distant metastasis occurs late during the genetic evolution of pancreatic cancer. Nature 467: 1114-1117, 2010.

27. Uesugi A, Kozaki K, Tsuruta T, et al: The tumor suppressive microRNA miR-218 targets the mTOR component Rictor and inhibits AKT phosphorylation in oral cancer. Cancer Res 71: 5765-5778, 2011.

28. Tatarano S, Chiyomaru T, Kawakami K, et al: $m i R-218$ on the genomic loss region of chromosome 4p15.31 functions as a tumor suppressor in bladder cancer. Int J Oncol 39: 13-21, 2011.

29. Leite KRM, Sousa-Canavez JM, Reis ST, et al: Change in expression of miR-let7c, miR-100, and miR-218 from high grade localized prostate cancer to metastasis. Urol Oncol 29: 265-269, 2011

30. Alajez NM, Lenarduzzi M, Ito E, et al: MiR-218 suppresses nasopharyngeal cancer progression through downregulation of survivin and the SLIT2-ROBO1 pathway. Cancer Res 71: 2381-2391, 2011

31. Gao CP, Zhang ZY, Liu WZ, Xiao SD, Gu WQ and Lu H: Reduced microRNA-218 expression is associated with high nuclear factor kappa B activation in gastric cancer. Cancer 116: 41-49, 2010.

32. Davidson MR, Larsen JE, Yang IA, et al: MicroRNA-218 is deleted and downregulated in lung squamous cell carcinoma. PLoS One 5: e12560, 2010.

33. Martinez I, Gardiner AS, Board KF, Monzon FA, Edwards RP and Khan SA: Human papillomavirus type 16 reduces the expression of microRNA-218 in cervical carcinoma cells. Oncogene 27 2575-2582,2008

34. Tie J, Pan YL, Zhao LN, et al: MiR-218 inhibits invasion and metastasis of gastric cancer by targeting the Robol receptor. PLoS Genet 6: e1000879, 2010.

35. Wu DW, Cheng YW, Wang J, Chen CY and Lee H: Paxillin predicts survival and relapse in non-small cell lung cancer by microRNA-218 targeting. Cancer Res 70: 10392-10401, 2010

36. Zhou XY, Chen XJ, Hu LM, et al: Polymorphisms involved in the miR-218-LAMB3 pathway and susceptibility of cervical cancer, a case-control study in Chinese women. Gynecol Oncol 117: 287-290, 2010.

37. Noel LS, Champion BR, Holley CL, et al: RoBo-1, a novel member of the urokinase plasminogen activator receptor/CD59/ Ly-6/snake toxin family selectively expressed in rat bone and growth plate cartilage. J Biol Chem 273: 3878-3883, 1998.

38. Ploug $M$ and Ellis V: Structure-function relationships in the receptor for urokinase-type plasminogen activator. Comparison to other members of the Ly-6 family and snake venom $\alpha$-neurotoxins. FEBS Lett 349: 163-168, 1994.

39. Yang L, Li Q, Wang Q, Jiang Z and Zhang L: Silencing of miRNA-218 promotes migration and invasion of breast cancer via Slit2-Robol pathway. Biomed Pharmacother 66: 535-540, 2012.

40. Woo S, Fish JE, Wythe JD, Bruneau BB, Stainier DY and Srivastava D: A novel Slit-Robo-miR-218 signaling axis regulates VEGF-mediated heart tube formation in zebrafish. The 69th Annual Meeting of the Society for Developmental Biology/ Japanese Society of Developmental Biologists. Dev Biol 344: 433, 2010 . 